\title{
Microplane MOdel For CONCRETE. I: STRESS-STRAin BOUNDARIES AND FinIte STRAIN
}

\author{
By Zdeněk P. Bažant, ${ }^{1}$ Fellow, ASCE, Yuyin Xiang, ${ }^{2}$ and Pere C. Prat, ${ }^{3}$ Member, ASCE
}

\begin{abstract}
The paper presents an improvement of the microplane model for concrete-a constitutive model in which the nonlinear triaxial behavior is characterized by relations between the stress and strain components on a microplane of any orientation under the constraint that the strains on the microplane are the projections of the macroscopic strain tensor. The improvement is achieved by a new concept: the stress-strain boundaries, which can never be exceeded. The advantage of this new concept is that various boundaries and the elastic behavior can be defined as a function of different variables (strain components). Thus, whereas for compression the stress-strain boundaries are defined on the microplanes separately for volumetric and deviatoric components, for tension an additional boundary is defined in terms of the total normal strains. This is necessary to achieve a realistic triaxial response at large tensile strains. For microplane shear, a friction law with cohesion is introduced. The present model is simpler than the previous microplane model. Finally, the microplane model is generalized to finite, but only moderately large, strains. Verification and calibration by test data are left to a subsequent companion paper.
\end{abstract}

\section{INTRODUCTION}

Although the last two decades have witnessed a major progress in the formulation of the nonlinear triaxial constitutive relations of brittle heterogeneous materials such as concrete, a fully realistic model has remained an elusive goal. The present paper presents a new formulation intended to make a major advance toward that goal. Among the various possible approaches, such as plasticity, continuum damage mechanics, fracturing theory, plastic-fracturing theory, or endochronic theory, a particularly powerful and versatile approach to concrete (as well as soils and other materials), which trades simplicity of concept for an increase of numerical work left to the computer, is the microplane model (Bažant 1984). This model represents a generalization of the initial idea of Taylor (1938), who proposed characterizing the constitutive behavior of polycrystalline metals by relations between the stress and strain vectors acting on planes of all possible orientations within the material and determining the macroscopic strain or stress tensors as a summation (or resultant) of all these vectors under the assumption of a static or kinematic constraint.

Taylor's idea was soon recognized as the most realistic way to describe the plasticity of metals, but the lack of computers in the early times prevented practical application. Batdorf and Budianski (1949) were first to extend Taylor's idea and develop a realistic model for plasticity of polycrystalline metals, still considered among the best. Many other researchers subsequently refined or modified this approach to metals (Kröner 1961; Budianski and Wu 1962; Lin and Ito 1965, 1966; Hill 1965, 1966; Rice 1970). Extensions for the hardening inelastic response of soils and rocks were also made (Zienkiewicz and Pande 1977; Pande and Sharma 1981, 1982; Pande and Xiong 1982).

In all the aforementioned models, it was assumed that the stress vector acting on various planes in the material, called the slip planes, was the projection of the macroscopic stress

\footnotetext{
'Walter P. Murphy Prof., of Civ. Engrg. and Mat. Sci., Northwestern Univ., Evanston, IL 60208.

${ }^{2}$ Grad. Res. Asst., Northwestern Univ., Evanston, IL.

${ }^{3}$ Visiting Scholar at Northwestern Univ., Assoc. Prof. on leave from Tech. Univ. of Catalunya, Barcelona, Spain.

Note. Associate Editor: Robert Y. Liang. Discussion open until August 1, 1996. Separate discussions should be submitted for the individual papers in this symposium. To extend the closing date one month, a written request must be filed with the ASCE Manager of Journals. The manuscript for this paper was submitted for review and possible publication on September 19, 1994. This paper is part of the Journal of Engineering Mechanics, Vol. 122, No. 3, March, 1996. OASCE, ISSN 0733-9399/96/ 0003-0245-0254/\$4.00 + \$.50 per page. Paper No. 9252.
}

tensor. As shown later (Bažant 1984), this constraint, which is a static constraint, prevents the model from being generalized for postpeak strain softening behavior or damage. It was realized that the extension to softening damage requires replacing the static constraint by a kinematic constraint, in which the strain vector on any inclined plane in the material is the projection of the macroscopic strain tensor (Bažant 1984). The kinematic constraint makes it possible to avoid spurious localization among orientations such that all the strain softening localizes preferentially into a plane of only one orientation.

In all applications to metals, the formulations emanating from Taylor's work and Batdorf and Budianski's work were called the slip theory of plasticity (in several applications that exist for rock, the term multilaminate model was used). The terms "slip theory," however, became unsuitable for the description of damage in quasi-brittle materials. For example, the inelastic behavior of concrete on the microscale does not physically represent slip, whether plastic or frictional (except under extremely high confining stresses), but mainly microfracturing. For this reason, the neutral term "microplane model," applicable to any physical type of inelastic behavior, was coined (Bažant 1984) (although a nondescriptive term such as "Taylor-Batdorf-Budianski model," perhaps with the names of further contributors, could also be used). The term "microplane" reflects the fact that the material properties are characterized by relations between the stress and strain components on planes of various orientations imagined to characterize the microstructure of the material. The tensorial invariance restrictions need not be directly enforced in the constitutive relations, which is a simplifying feature of the microplane formulation. They are automatically satisfied by superimposing in a suitable manner the responses from the microplanes of all orientations. As introduced for the microplane model (Bažant 1984), this is done by means of a variational principle (principle of virtual work).

The present study focuses on concrete. The microplane model of concrete was first devised for tensile fracturing ( $\mathrm{Ba}$ zant and Oh 1983, 1985; Bažant and Gambarova 1984), and later for nonlinear triaxial behavior in compression with shear (Bažant and Prat 1988a, b). These new models differed from the previous models by using the kinematic (rather than static) constraint for the microplanes (Bažant 1984). Because the tangential material stiffness matrix for these models may lose positive definiteness (due to postpeak strain softening as well as lack of normality), a nonlocal generalization was developed to prevent spurious excessive localization of damage in structures and spurious mesh sensitivity (Bažant and Ožbolt 1990; Ožbolt and Bažant 1992). An explicit formulation and efficient 
numerical algorithm for the microplane model of Bažant and Prat (1988a, b) was recently presented by Carol et al. (1992). It was also shown that the microplane model with a kinematic constraint can be cast in the form of continuum damage mechanics in which the damage variable, representing the reduction of the stress-resisting cross-section area fraction in the material, is a fourth-order tensor, independent of microscopic material stiffness characteristics (Carol et al. 1991). An efficient implementation of the microplane model for concrete in dynamic explicit finite element programs was proposed by Cofer (1992) and Cofer and Kohut (1994), for both coarse and fine meshes.

Although the microplane model of Bažant and Prat (1988a, b) was initially thought to perform adequately for postpeak softening damage in both compression and tension, Jirásek (1993) found from numerical experience that, in postpeak uniaxial tension, excessively large positive lateral strains develop at large tensile strains. He then showed that this unrealistic behavior was caused by localization of tensile strain softening into the volumetric strain, while the deviatoric strains on the strain softening microplanes exhibited unloading. It was recognized that this localization of tensile softening damage into one of the two normal strain components in tension (that is, the volumetric one) was an inevitable consequence of separation of normal strains into the volumetric and deviatoric parts. However, this separation was previously shown necessary (Bažant and Prat 1988a, b) for correct modeling of triaxial behavior in compression as well as for achieving the correct elastic Poisson's ratio. The problem was first remedied by removing tensile strain softening from the model of Bažant and Prat (1988a, b) and coupling this model in series with that of Bažant and Oh (1983, 1985), which describes tensile strain softening but behaves elastically in compression. However, such a remedy seems an artifice. Also, it does not allow an explicit algorithm and is computationally inefficient.

A better remedy, free of these shortcomings, will be presented here, based on the new idea of stress-strain boundaries (Bažant 1993) proposed at a recent conference (Bažant et al. 1994a). The formulation will also be extended to finite strains, heeding all the restrictions of continuum mechanics of solids. Verification and calibration by test data are relegated to a subsequent companion paper (Bažant et al. 1996), in which it will be attempted to develop a systematic approach to the identification of material parameters and to filter out from the test data the effects of postpeak strain localization in test specimens and the size effects.

\section{MICROPLANE FORMULATION APPLICABLE TO CONCRETE}

In the classical approach, the constitutive model is defined by algebraic or differential relations between the components $\sigma_{i j}$ and $\varepsilon_{i j}$ of the stress and strain tensors, based on the theory of tensorial invariants. In the microplane approach, the constitutive model is defined by a relation between the stresses and strains acting on a plane of arbitrary orientation within the material. The orientation of this plane, called the microplane, is characterized by the unit normal $n$ of components $n_{i}$ (indices $i$ and $j$ refer to the components in Cartesian coordinates $x_{i}$ ). The basic hypothesis, which makes it possible to describe strain softening in a stable manner (Bažant 1984), is that the strain vector $\varepsilon_{N}$ on the microplane [Fig. 1(a)] is the projection of the macroscopic strain tensor $\varepsilon_{i j}$. So the components of this vector are $\varepsilon_{N_{i}}=\varepsilon_{i j} n_{j}$. The normal strain on the microplane is $\varepsilon_{N}=n_{i} \varepsilon_{N_{i}}$, that is

$$
\varepsilon_{N}=N_{i j} \varepsilon_{i j} ; \quad N_{i j}=n_{i} n_{j}
$$

where repeated indices imply summation over $i=1,2,3$. The

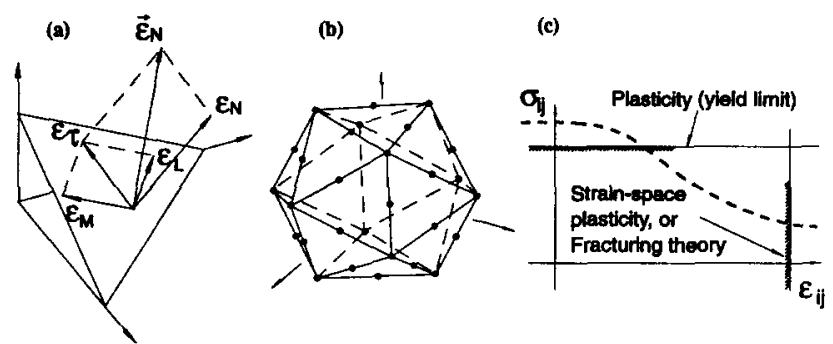

FIG. 1. (a) Strain Components on General Microplane; (b) Directions of Microplane Normals (Circles) for System of 21 Microplanes per Hemlsphere; (c) Classical Tensorial Theories and Present Theory

mean normal strain, called the volumetric strain $\varepsilon_{V}$, and the deviatoric strain $\varepsilon_{D}$ on the microplane, are defined as follows:

$$
\varepsilon_{V}=\varepsilon_{k k} / 3 ; \quad \varepsilon_{D}=\varepsilon_{N}-\varepsilon_{V}
$$

To characterize the shear strains on the microplane [Fig. 1(a)], we need to define two coordinate directions $M$ and $L$, given by two orthogonal unit coordinate vectors $m$ and $l$ of components $m_{i}$ and $l_{l}$ lying within the microplane. We may for example choose vector $m_{i}$ to be normal to axis $x_{3}$, in which case $m_{1}=n_{2}\left(n_{1}^{2}+n_{2}^{2}\right)^{-1 / 2}, m_{2}=-n_{1}\left(n_{1}^{2}+n_{2}^{2}\right)^{-1 / 2}$, and $m_{3}=0$, but $m_{1}=1$ and $m_{2}=m_{3}=0$ if $n_{1}=n_{2}=0$. Alternatively, to get a vector $m_{i}$ normal to axis $x_{1}$ or axis $x_{2}$, we carry out permutations $123 \rightarrow 231 \rightarrow 312$ of the indices in the preceding formulas. The other coordinate vector $l_{l}$ within the microplane is obtained as the vector product, $\mathbf{l}=\mathbf{m} \times \mathbf{n}$. To minimize directional bias of $\mathbf{m}$ and $\mathbf{l}$ among microplanes, we alternate among choosing vectors $\mathrm{m}$ to be normal to axis $x_{1}, x_{2}$, or $x_{3}$.

The magnitudes of the shear strain components on the microplane in the directions of $\mathbf{m}$ and $\mathbf{l}$ are $\varepsilon_{M}=m_{i}\left(\varepsilon_{i j} n_{j}\right)$ and $\varepsilon_{L}$ $=l_{l}\left(\varepsilon_{i j} n_{j}\right)$. Because of symmetry of tensor $\varepsilon_{i j}$, the shear strain components may be written as follows (Bažant and Prat 1988):

$$
\varepsilon_{M}=M_{i j} \varepsilon_{i j} ; \quad \varepsilon_{L}=L_{j} \varepsilon_{i j}
$$

in which the following symmetric tensors were introduced:

$$
M_{i j}=\left(m_{i} n_{j}+m_{j} n_{i}\right) / 2 ; \quad L_{i}=\left(l_{i} n_{j}+l_{j} n_{l}\right) / 2
$$

The stress components on the microplane cannot in general be equal to the projections of the macroscopic stress tensor $\sigma_{i j}$ if the strains represent the projections of $\varepsilon_{i j}$. Thus, static equivalence or equilibrium between the macrolevels and microlevels must be enforced by other means. To this end, consider now a small representative volume of the material, given by a small cube of side $\Delta h$. A pair of two parallel sides corresponds to a microplane labeled by subscript $N$, and the other two pairs of parallel sides correspond to orthogonal microplanes labeled by subscripts $P$ and $Q$. The strain vectors on these microplanes may be assumed to have the meaning defined by $\Delta \mathbf{u}_{N} / \Delta h=$ $\varepsilon_{N}, \Delta \mathbf{u}_{P} / \Delta h=\varepsilon_{P}$, and $\Delta \mathbf{u}_{Q} / \Delta h=\Delta \varepsilon_{Q}$, in which $\Delta \mathbf{u}_{N}, \Delta \mathbf{u}_{P}$, and $\Delta \mathbf{u}_{Q}$ are the differences in the displacement vector between the opposite sides of the cube in the directions labeled by $N, P$, and $Q$. The equality of the incremental virtual work of stresses within the representative volume on the macrolevel and the work of stresses on the three microplanes representing the sides of the cube implies that $\Delta h^{3} \sigma_{u} \delta \varepsilon_{i j}=\Delta h^{2}\left(\sigma_{N} \cdot \delta \Delta \mathbf{u}_{N}+\right.$ $\sigma_{p} \cdot \delta \Delta u_{P}+\sigma_{Q} \cdot \delta \Delta u_{Q}$ ), where $\delta$ denotes the variations. The strain vectors $\varepsilon_{N}, \varepsilon_{P}$, and $\varepsilon_{Q}$ include the contributions of elastic deformations as well as displacements due to cracking (and possibly also to frictional plastic slip). The cracking or other inelastic deformation happens randomly on planes of various orientations within the material, and the macroscopic continuum must represent these strains statistically, in the average sense. Therefore 


$$
\Delta h^{3} \sigma_{v} \delta \varepsilon_{v}=\frac{\Delta h^{2}}{2 \pi} \int_{\Omega}\left(\sigma_{N} \cdot \delta \Delta u_{N}+\sigma_{P} \cdot \delta \Delta u_{P}+\sigma_{Q} \cdot \delta \Delta u_{Q}\right) d \Omega
$$

in which the integral represents averaging over all spatial orientations; $d \Omega=\sin \theta d \theta d \phi$, where $\theta$ and $\phi=$ spherical angles and $\boldsymbol{\Omega}=$ surface of a unit hemisphere (whose surface area is $2 \pi)$; and $\sigma_{N}=\left(\sigma_{N}, \sigma_{M}, \sigma_{L}\right), \varepsilon_{N}=\left(\varepsilon_{N}, \varepsilon_{M}, \varepsilon_{L}\right)$, etc. Now, obviously, $\int_{\Omega} \sigma_{N} \cdot \delta \Delta u_{N} d \Omega=\int_{\Omega} \sigma_{P} \cdot \delta \Delta u_{P} d \Omega=\int_{\Omega} \sigma_{Q} \cdot \Delta u_{Q} d \Omega$. Consequently, the variational equation (5) becomes

$$
\frac{2 \pi}{3} \sigma_{i j} \delta \varepsilon_{j}=\int_{\Omega} \sigma_{N} \cdot \delta \varepsilon_{N} d \Omega=\int_{\Omega}\left(\sigma_{N} \delta \varepsilon_{N}+\sigma_{M} \delta \varepsilon_{M}+\sigma_{L} \delta \varepsilon_{L}\right) d \Omega
$$

This equation was introduced in Bažant (1984) directly, as the condition that, for any variation $\delta \varepsilon_{i}$, the virtual work of macrostresses within a unit sphere must be equal to the virtual work of microstresses representing tractions on the surface elements of the sphere.

According to (1) and (4), $\delta \varepsilon_{N}=N_{v} \delta \varepsilon_{i j}, \delta \varepsilon_{M}=M_{i} \delta \varepsilon_{i}$, and $\delta \varepsilon_{L}=L_{v} \delta \varepsilon_{i j}$. Substituting this into (6), we obtain

$$
\left(\frac{4 \pi}{3} \sigma_{i j}-2 \int_{\Omega} s_{v} d \Omega\right) \delta \varepsilon_{i j}=0
$$

in which we introduce the notation

$$
s_{u}=\sigma_{N} N_{v}+\sigma_{M} M_{i}+\sigma_{L} L_{i j}
$$

Since (7) is a variational equation, which must be satisfied for any variation $\delta \varepsilon_{i j}$, the expression in parentheses must vanish. This yields

$$
\sigma_{v}=\frac{3}{2 \pi} \int_{\Omega} s_{v} d \Omega \approx 6 \sum_{\mu=1}^{N_{m}} w_{\mu} s_{v}^{(\mu)}
$$

in which $\Sigma_{\mu} w_{\mu}=0.5$ per hemisphere and the last expression represents an approximate numerical evaluation of the integral over the hemisphere; subscripts $\mu$ refer to a chosen set of integration points representing orientations defined by unit vectors $n_{i}^{(\mu)}$ and shown by the circled points in Fig. 1(b); $w_{\mu}$ $=$ integration weights associated with these points; and superscripts $(\mu)$ label the values corresponding to these directions. While the integral over $\Omega$ represents integration over an infinite number of microplanes, the discrete approximation represents summation over a finite number of microplanes. The flow of calculation between the macrolevel and microlevel is explained by Fig. 2.

Formulation of an optimal numerical integration formula over the surface of a hemisphere is not a trivial matter. The problem has been studied extensively by mathematicians, and Gaussian integration formulas of various degrees of approximation have been developed. One sufficiently accurate formula, which consists of 28 microplanes (i.e., 28 integration points) over a hemisphere, is given by Stroud (1971). A more efficient and only slightly less accurate formula, involving 21 microplanes, was developed by Bažant and Oh (1986), who also studied the accuracy of various formulas in terms of representation of postpeak strain softening, for which the errors of numerical integration get manifested most. The orientations of the normals to the microplanes in the 21-point formula (which evaluates the integral exactly for all polynomials up to degree 9) represent the radial directions to the vertices and centers of the edges of a regular icosahedron, shown in Fig. 1(b). Fewer than 21 microplanes cannot give sufficient accuracy (Bažant and Oh 1985). To write an efficient computer program, the values of $N_{i j}^{(\mu)}, M_{i}^{(\mu)}$, and $L_{i j}^{(\mu)}$ should be calculated in advance for all the microplanes and stored in memory. The values of $w_{\mu}$ and $n_{i}^{(\mu)}$ must also be stored in advance.

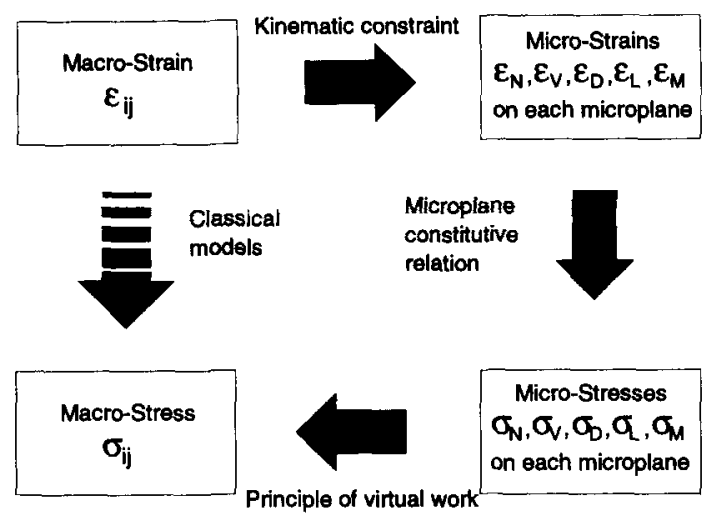

FIG. 2. Flow of Calculation In Microplane Model (Present Paper) and In Classical Models

\section{MICROPLANE STRESSES AND VOLUMETRIC- DEVIATORIC SPLIT}

Calculation of the microplane stresses in (8) from the microplane strains is a problem of constitutive modeling, which we now address. Material isotropy will be ensured in two ways: by using a uniform unit weight, independent of orientation $n_{i}$, in the integral over $\Omega$ in (9); and by using the same stress strain relation for microplanes of all orientation $n_{i}$. The elastic responses of the material modeled by the microplane system may be characterized on the microplane level in the rate form as follows:

$$
\begin{array}{ll}
\dot{\sigma}_{V}=E_{V} \dot{\varepsilon}_{V} ; & \dot{\sigma}_{D}=E_{D} \dot{\varepsilon}_{D} \\
\dot{\sigma}_{M}=E_{T} \dot{\varepsilon}_{M} ; & \dot{\sigma}_{L}=E_{T} \dot{\varepsilon}_{L}
\end{array}
$$

Note that the relation $\dot{\sigma}_{N}=E_{N} \dot{\varepsilon}_{N}$ is valid only if $E_{V}=E_{D} . E_{V}$, $E_{D}$, and $E_{T}=$ elastic moduli on the microplane level, same for all the microplanes. As shown in Bažant and Prat (1988a, b), the microplane moduli are related to the macroscopic Young's modulus $E$ and Poisson's ratio $v$ as follows

$$
E_{V}=\frac{E}{1-2 \nu} ; \quad E_{D}=\frac{5 E}{(2+3 \mu)(1+\nu)} ; \quad E_{T}=\mu E_{D} \quad(11 a-c)
$$

in which $\mu=$ a parameter that may be chosen or can be optimized so as to match the given test data best. Bažant and Prat $(1988 \mathrm{a}, \mathrm{b})$ stated relations equivalent to (11), using parameter $\eta=E_{D} / E_{V}$ instead of $\mu$. They also found the range of $\eta$-values giving optimum fits of test data for concrete, and this range corresponds to $\mu$-values close to 1 . Therefore, for the present calculations, we choose $\mu=1$, or $E_{T}=E_{D}$. As transpires from Carol et al. (1991), the value $\mu=1$ is also comceptually appealing, because it makes it possible to characterize damage by a fourth-rank tensor that is independent of the material stiffness properties. This will be discussed further. (Another special case, which, however, is not realistic for concrete, is $E_{V}=E_{D}$ or $\mu=(1-4 v) /(1+v)$; in that case $\sigma_{N}=$ $E_{N} \varepsilon_{N}$, with $E_{N}=E_{V}$, which corresponds to the case of no volumetric-deviatoric split.)

One reason for splitting the normal strain at the microplane into the volumetric and deviatoric normal components is that a general model ought to be capable of giving any thermodynamically admissible Poisson's ratio (between 0.5 and -1 ). That this is indeed so can be checked by eliminating $\mu$ from (11) and solving for $\nu$, which yields $\nu=\left(5 E_{V}-2 E_{D}-3 E_{T}\right) /$ $\left(10 E_{V}+2 E_{D}+3 E_{T}\right)$. This equation shows that it is possible to obtain any thermodynamically admissible value of Poisson's ratio; that is, $-1 \leq v \leq 0.5$. From this equation it is further clear that, for the case of no split, which corresponds to the case $E_{V}=E_{D}=E_{N}$, one would have $v=\left(3 E_{N}-3 E_{T}\right) /$ $\left(12 E_{N}+3 E_{T}\right)$, which implies that the Poisson's ratio would 

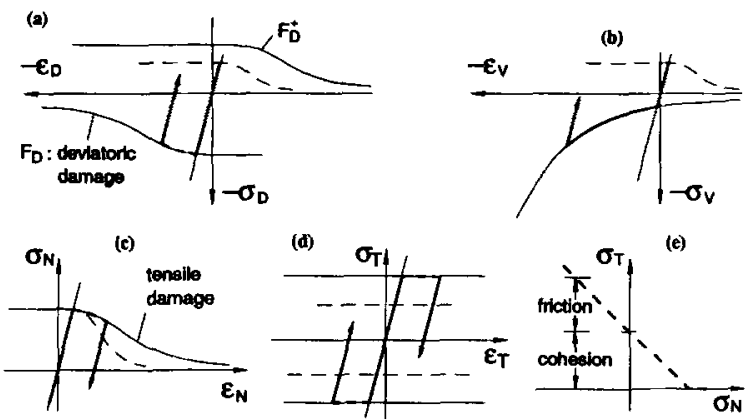

FIG. 3. (a-d) Stress-Strain Boundarles for Microplane; (e) Friction Law for Microplane

be restricted to the range from 0.25 to -1 . Although this range would suffice for concrete, the microplane model in principle could not be realistic if it were restricted to Poisson's ratios less than 0.25 .

It may also be noted that if the shear stiffness is neglected $\left(E_{T}=0\right.$ or $\mu=0$ ) and no volumetric-deviatoric split is used $\left(\sigma_{N}=E_{N} \varepsilon_{N}\right)$, which was assumed in the initial model of Bažant and Oh $(1983,1985)$ for tensile fracturing, the Poisson's ratio is restricted to the value $\nu=0.25$. However, such a restriction is not realistic, and besides, the elastic as well as inelastic shear strain on the microplane level appears to be important for correct modeling of the effect of confining pressure on compression failure.

In analogy to multiaxial Hooke's law, which may be written as $\sigma_{11}=E^{\prime}\left(\varepsilon_{11}+\nu^{\prime} \varepsilon_{22}+\nu^{\prime} \varepsilon_{33}\right)$ (where $E^{\prime}$ and $\nu^{\prime}=$ constants), one could introduce for each microplane the relation $\sigma_{N}=$ $E_{N}\left(\varepsilon_{N}+\nu^{\prime} \varepsilon_{N_{M}}+\nu^{\prime} \varepsilon_{N_{L}}\right)$. This could be written as $\sigma_{N}=E_{N}\left(\varepsilon_{N}\right.$ $\left.+\nu^{\prime} \varepsilon_{\text {lat }}\right)$, in which $\varepsilon_{\text {lat }}=\left(\varepsilon_{N_{M}}+\varepsilon_{N_{L}}\right) / 2=\left(2 \varepsilon_{v}-\varepsilon_{N}\right) / 2=\varepsilon_{V}-$ $\left(\varepsilon_{D} / 2\right)$; here, $\varepsilon_{\text {lat, }}$, called the lateral strain (Hasegawa and Bažant 1993), is invariant with respect to rotations of the $K$ and $M$ directions about normal $N$. At first one might think that the lateral strain might be the most appropriate variable to describe the confinement effect in compression loading on the microplane level; however, from the foregoing relations it is clear that this is really equivalent to formulating the inelastic behavior on the microplane in terms of $\varepsilon_{D}$ and $\varepsilon_{V}$, which is simpler.

The main reason for the volumetric-deviatoric split with independent moduli $E_{V}$ and $E_{D}$ (Bažant and Prat 1988a, b) is the absence of stress peak for the hydrostatic compression test and the uniaxial-strain compression test [see the tests of Bažant et al. (1986)], while at the same time the loading by uniaxial compressive stress or other compressive loading with uninhibited volume expansion exhibits a stress peak followed by postpeak strain softening. Without the aforementioned split, compressive loading with restricted volume expansion (hydrostatic compression and uniaxial strain) would, incorrectly, exhibit a peak stress and postpeak strain softening.

In the initial proposal of microplane model with strain softening (Bažant 1984), the stress-strain relation for the normal and shear components of stresses and strains of the microplanes had the form of flow rule of incremental plasticity, based on subsequent yield surfaces and loading potentials for the microplane. However, subsequent studies have shown that this was unnecessarily complicated. As it turned out (Bažant and Prat 1988a, b), one can assume, for the case of virgin loading, a total algebraic stress-strain relation on the microplane level; that is, $\sigma_{V}, \sigma_{D}, \sigma_{M}$, and $\sigma_{L}$ can be assumed to be functions of $\varepsilon_{V}, \varepsilon_{D}, \varepsilon_{M}$, and $\varepsilon_{L}$. Further, it turned out that each stress component can be considered to depend only on the associated strain component, with the exception of shear stress $\sigma_{M}$ (or $\sigma_{L}$ ), which must depend not only on $\varepsilon_{M}$ (or $\varepsilon_{L}$ ), but also on $\sigma_{V}$. Without this cross dependence, which reflects in- ternal friction, it is not possible to model standard triaxial tests at high confining pressures.

\section{NEW CONCEPT: STRESS-STRAIN BOUNDARIES}

The main reason for the new concept of stress-strain boundaries is the modeling of triaxial behavior in tension. But there are further reasons. In the previous microplane model for compressive triaxial behavior (Bažant and Prat 1988a, b), the stress-strain relations for the microplane were smooth curves. However, difficulties arose in the handling of the transition from reloading to virgin inelastic loading in the quadrants of negative stress-strain ratio, and complicated rules were needed (Hasegawa and Bažant 1993; Ožbolt and Bažant 1992). Also, the modeling of cyclic loading was more difficult. These difficulties, too, are avoided by the new concept.

The condition that the response may not exceed the specified boundary curve $\sigma_{N}=f_{N}\left(\varepsilon_{N}, \sigma_{V}\right)$ makes it easy to ensure continuity at the transition from elastic behavior, defined separately for volumetric and deviatoric components, and the strain-softening damage behavior in tension, defined without the volumetric-deviatoric split (Bažant 1993). It would be too complicated to devise stress-strain relations that would describe such transitions without any discontinuity.

The stress-strain boundaries, shown in Fig. 3, are defined as follows:

$$
\begin{gathered}
\sigma_{N}=F_{N}\left(\varepsilon_{N}, \sigma_{V}\right) ; \quad \sigma_{V}=-F_{V}\left(-\varepsilon_{V}\right) ; \quad \sigma_{T}=F_{T}\left(\sigma_{N}\right) \\
\sigma_{D}=-F_{D}\left(-\varepsilon_{D}\right) ; \quad \sigma_{D}=F_{D}^{+}\left(\varepsilon_{D}\right)
\end{gathered}
$$

in which $\sigma_{T}$ stands for either $\sigma_{M}$ or $\sigma_{L}$. The reason for writing the minus signs is that functions $F_{N}, F_{V}, F_{D}, F_{D}^{+}$, and $F_{T}$ are defined as positive-valued functions of positive arguments. Function $F_{T}$ defines only the boundary for positive stresses [Fig. 3(b)], and the other boundary for negative stresses is symmetric. The dependence of $\sigma_{T}$ on $\sigma_{N}$ characterizes friction on the microplane, as well as the fact that a widely opened rough crack offers less resistance to shear than a narrow rough crack. The boundary for compressive $\sigma_{D}$ is provided by $F_{D}$ (for $\varepsilon_{D}<0$ ). The tensile boundary $F_{N}$ for $\varepsilon_{N}>0$ does not suffice to prevent tensile $\varepsilon_{D}$ from becoming unreasonably large (particularly in triaxial tests at very high pressures), and therefore an additional tensile boundary for $\sigma_{D}$ needs to be provided by $F_{D}^{+}$.

Note that despite path independence on the microplane level, the macroscopic material response to various nonproportional loading paths is path dependent. This is because many combinations of loading and unloading are possible in each loading step.

Experience shows that, for sufficient accuracy, a system of at least 21 microplanes must be associated with each integration point of each finite element [Bažant and Oh $(1985,1986)$; used in Bažant and Ožbolt (1990)]. The number, however, can be reduced in the case symmetries such as plane stress, plane strain, or uniaxial stress. In each loading step, an explicit computational algorithm can be formulated as follows. First the new values of macrostrains $\varepsilon_{i j}$ are calculated at each integration point from the new (incremented) values of nodal displacements. Then, for each integration point, the new values of $\varepsilon_{N}$, $\varepsilon_{V}, \varepsilon_{D}, \varepsilon_{M}$, and $\varepsilon_{L}$ are calculated for all the microplanes from (1)-(3). Using these values, the following new stress values are calculated for each microplane:

$$
\begin{aligned}
\sigma_{V}^{e} & =\sigma_{V}^{i}+E_{V}\left(\varepsilon_{V}-\varepsilon_{V}^{i}\right) ; \\
\sigma_{D}^{e} & =\sigma_{D}^{i}+E_{D}\left(\varepsilon_{D}-\varepsilon_{D}^{i}\right) ; \quad \sigma_{N}^{e}=\sigma_{V}^{e}+\sigma_{D}^{e} \\
\sigma_{M}^{e} & =\sigma_{M}^{i}+E_{T}\left(\varepsilon_{M}-\varepsilon_{M}^{i}\right) ; \quad \sigma_{L}^{e}=\sigma_{L}^{i}+E_{T}\left(\varepsilon_{L}-\varepsilon_{L}^{\prime}\right) ; \\
\sigma_{V}^{\prime} & =\max \left[\sigma_{V}^{e},-F_{V}\left(\varepsilon_{V}\right)\right]
\end{aligned}
$$



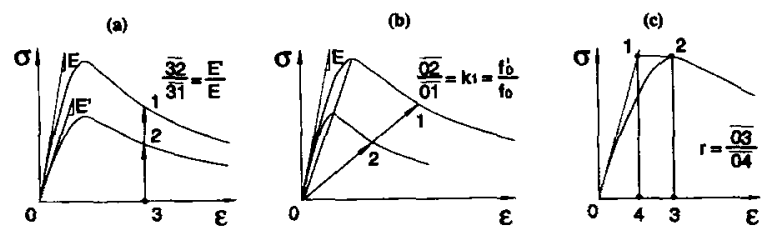

FIG. 4. (a-b) Vertical and Radial Scalling (Affinity Transformations) of Stress-Strain Curves; (c) Meaning of Ductillty $r$

$$
\begin{gathered}
\sigma_{N}^{\prime}=\sigma_{V}^{\prime}+\max \left[\sigma_{D}^{e},-F_{D}\left(\left\langle-\varepsilon_{D}\right\rangle\right)\right] ; \\
\sigma_{N}=\min \left[\sigma_{N}^{\prime}, F_{N}\left(\left\langle\varepsilon_{N}\right\rangle, \hat{\sigma}_{V}\right)\right]
\end{gathered}
$$

Superscripts $i$ denote the previously calculated initial values at the beginning of the loading step, and absence of a superscript means the new values; superscripts $e$ denote the elastically calculated new stress values; $\langle x\rangle=\max (x, 0)=$ positive part of $x$ (this symbol, called the Macauley bracket, is used so that functions $F_{N}, \ldots, F_{T}$ could be defined for only the positive values of strain arguments); and $\hat{\sigma}_{v}=\sigma_{v}^{i}$, but if the load step is iterated it helps accuracy to take $\hat{\sigma}_{v}$ as the value of $\sigma_{V}$ obtained in the previous iteration. After sweeping through all the microplanes $\mu=1, \ldots, N_{m}$, one evaluates

$$
\bar{\sigma}_{V}=\sum_{\mu=1}^{N_{m}} w_{\mu} \sigma_{N}^{(\mu)}
$$

Then, for each microplane one can calculate

$$
\sigma_{v}=\min \left(\sigma_{v}^{\prime}, \bar{\sigma}_{v}\right)
$$

For $\varepsilon_{V}-\varepsilon_{V}^{i}>0$

$$
\sigma_{T}^{\prime}=\min \left[\sigma_{T}^{e}, F_{T}\left(\sigma_{N}\right)\right] ; \quad \sigma_{T}=\max \left[\sigma_{T}^{\prime},-F_{T}\left(\sigma_{N}\right)\right]
$$

For $\varepsilon_{v}-\varepsilon_{V}^{i} \leq 0$

$$
\sigma_{T}^{\prime}=\max \left[\sigma_{T}^{e},-F_{T}\left(\sigma_{N}\right)\right] ; \quad \sigma_{T}=\min \left[\sigma_{T}^{\prime}, F_{T}\left(\sigma_{N}\right)\right]
$$

After sweeping again through all the microplanes, all the new values of the microplane stresses at the end of the loading step are known, and the macrostresses can then be calculated from (8)-(9). The inelastic parts of the new macrostresses must subsequently be modified according to a suitable nonlocal formulation (unless the crack band approximation is used). But this subject is beyond the scope of the present paper.

Note that, except for $\hat{\sigma}_{v}$, the foregoing algorithm gives the new stresses as explicit functions of the new strains. No equations need to be solved. This is important for computational efficiency. Experience shows that taking $\hat{\sigma}_{V}=\sigma_{V}^{\prime}$ is normally sufficient for good accuracy, and then no iterations are needed and all the stress calculations are explicit.

The stress-strain boundary may be regarded as a strain-dependent yield limit. Such an idea could hardly be introduced in the classical macroscopic formulation of plasticity (based on stress invariants), because the boundary would be a surface in an 18-dimensional space of all $\sigma_{i j}$ and $\varepsilon_{i j}$ components. The microplane concept makes the idea of strain-dependent yield limit feasible - simple, in fact-because there are only a few stress and strain components on the microplane level. The strain-dependent yield limit may be illustrated by the curve in Fig. 1(c). The classical (stress space) plasticity is in this figure represented by the horizontal line for the yield limit. Now note that plastic metals have also been satisfactorily described by strain-space plasticity, which corresponds to the vertical line in this figure. Obviously a general curve should allow a better description because it is a combination of stress-space and strain-space plasticity theories.

\section{CONSTITUTIVE CHARACTERIZATION OF MATERIAL ON MICROPLANE LEVEL}

By fitting of various types of test data for concrete, the following functions, characterizing the constitutive properties of the material, have been identified:

$$
\begin{gathered}
F_{V}\left(-\varepsilon_{V}\right)=f_{V}^{0} \exp \left(-\frac{\varepsilon_{V}}{k_{1} k_{S}}\right) ; \quad f_{V}^{0}=E k_{1} k_{4} \\
F_{D}\left(-\varepsilon_{D}\right)=f_{D}^{0}\left(1-\frac{\varepsilon_{D}}{k_{1} c_{2}}\right)^{-1} ; f_{D}^{0}=E k_{1} c_{4} \quad\left(\varepsilon_{D} \leq 0\right) \\
F_{D}^{+}\left(\varepsilon_{D}\right)=c_{S} f_{D}^{0}\left(1-\frac{\varepsilon_{D}}{k_{1} c_{2} c_{S}}\right)^{-1} \quad\left(\varepsilon_{D} \geq 0\right) \\
F_{N}\left(\varepsilon_{N}, \sigma_{V}\right)=f_{N}^{0}\left[1+\left(\frac{\varepsilon_{N}}{c k_{1}}\right)^{2}\right]^{-1} ; \\
c=c_{1}+\left\langle\frac{-c_{3} \sigma_{V}}{E_{V}}\right\rangle ; \quad f_{N}^{0}=E k_{1} \quad\left(\varepsilon_{N} \geq 0\right) \\
F_{T}\left(\sigma_{N}\right)=\left\langle E k_{1} k_{2}-k_{3} \sigma_{N}\right\rangle
\end{gathered}
$$

in which $k_{1}, \ldots, k_{5}=$ adjustable empirical constants, which take different values for different types of concretes; and $c_{1}, \ldots, c_{5}=$ fixed empirical constants that can be kept the same for all normal concretes. They have the values $c_{1}=5$, $c_{2}=6, c_{3}=50, c_{4}=130$, and $c_{5}=6$ [among the data fitted in the companion paper (Bažant et al. 1996), parameter $c_{5}$ affects only the fit of the standard triaxial tests at very high pressures]. In the absence of sufficient test data, it is recommended that the adjustable parameters be taken with the following reference values: $k_{1}=72 \times 10^{-6}, k_{2}=0.1, k_{3}=0.05, k_{4}=15$, and $k_{5}=150$. The value of Poisson's ratio has been considered $v$ $=0.18$. Except for $E$, all the parameters are dimensionless.

The macroscopic Young's modulus is a parameter whose change causes a vertical scaling transformation (affinity transformation) of all the response stress-strain curves. If this parameter is changed from $E$ to some other value $E^{\prime}$, all the stresses are multiplied by the ratio $E^{\prime} / E$ at no change of strains [Fig. 4(a)]. Parameter $k_{1}$ describes radial scaling transformation with respect to the origin. If this parameter is changed from $k_{1}$ to some other value $k_{1}^{\prime}$, all the stresses and all the strains are multiplied by the ratio $k_{1}^{\prime} / k_{1}$ [Fig. 4(b)].

The aforementioned reference values of material parameters, along with $E=58,000 \mathrm{MPa}$, yield the uniaxial compression strength $f_{\mathrm{c}}^{\prime}=42.4 \mathrm{MPa}$, as calculated by simulating the uniaxial compression test by incremental loading. The strain corresponding to the stress peak has been found to be $\varepsilon_{p}=$ 0.0022 . Now, if the user needs a microplane model that yields the uniaxial compressive strength $f_{c}^{*}$ and the corresponding strain at peak $\varepsilon_{p}^{*}$, one only needs to replace the reference values of parameters $k_{1}$ and $E$ by the following values:

$$
k_{1}^{*}=k_{1} \frac{\varepsilon_{p}^{*}}{\varepsilon_{p}} ; \quad E^{*}=E \frac{f_{c}^{*}}{f_{c}^{\prime}} \frac{\varepsilon_{p}}{\varepsilon_{p}^{*}}
$$

$(24 a, b)$

Table 1 shows the values of $f_{c}^{\prime}$ and $f_{t}^{\prime}$ for some typical values of material parameters (" $R$ " in Table 1 refers to the reference values stated above). It also gives the corresponding ductility, which is defined as $r=\varepsilon_{p} E / f^{\prime}$ and represents the ratio $\overline{03 / 04}$ in Fig. 4(c) $\left(f^{\prime}\right.$ is $f_{c}^{\prime}$ or $\left.f_{i}^{\prime}\right)$. The smaller $r$, the steeper the post-peak softening. The transformations according to (24) do not change the ratio $r$.

The aforementioned reference values of material parameters yield the following ratios:

$$
\frac{f_{t}^{\prime}}{f_{c}^{\prime}}=0.082 ; \quad \frac{f_{b c}^{\prime}}{f_{c}^{\prime}}=1.17 ; \frac{f_{c}^{s}}{f_{c}^{\prime}}=0.069 ; \quad \frac{\sigma_{r}}{f_{c}^{\prime}}=0.07 ; \quad \frac{\tau_{r}}{f_{c}^{s}}=0.3
$$


TABLE 1. Strength, Ductility, and Typical Material Parameters

\begin{tabular}{|c|c|c|c|c|c|c|c|c|c|c|}
\hline $\begin{array}{c}\text { Tests } \\
(1) \\
\end{array}$ & $\begin{array}{c}E \\
(2) \\
\end{array}$ & $\begin{array}{c}k_{1}\left(10^{-1}\right) \\
(3)\end{array}$ & $\begin{array}{r}k_{2} \\
(4) \\
\end{array}$ & $\begin{array}{r}k_{3} \\
(5) \\
\end{array}$ & $\begin{array}{l}k_{4} \\
(6) \\
\end{array}$ & $\begin{array}{l}k_{8} \\
(7)\end{array}$ & $\begin{array}{r}f_{c}^{\prime} \\
(8) \\
\end{array}$ & $\begin{array}{r}r_{c} \\
(9) \\
\end{array}$ & $\begin{array}{c}f_{t}^{\prime} \\
(10)\end{array}$ & $\begin{array}{r}r_{t} \\
(11) \\
\end{array}$ \\
\hline $\begin{array}{l}\text { Hognestad } \\
\text { van Mier } \\
\text { Petersson } \\
\text { Bažant } \\
\text { Green } \\
\text { Balmer } \\
\text { Bresler } \\
\text { Kupfer } \\
\text { Launay } \\
\text { Sinha }\end{array}$ & $\begin{array}{r}8,200 \\
58,000 \\
60,000 \\
6,000 \\
5,100 \\
3,500 \\
5,600 \\
5,500 \\
5,100 \\
4,000\end{array}$ & $\begin{array}{r}59 \\
58 \\
75 \\
117 \\
135 \\
65 \\
80 \\
56 \\
40 \\
60\end{array}$ & $\begin{array}{c}\mathrm{R} \\
0.6 \\
\mathrm{R} \\
\mathrm{R} \\
\mathrm{R} \\
\mathrm{R} \\
0.2 \\
\mathrm{R} \\
\mathrm{R} \\
0.6\end{array}$ & $\begin{array}{c}\mathrm{R} \\
0.12 \\
0.4 \\
\mathrm{R} \\
\mathrm{R} \\
0.4 \\
\mathrm{R} \\
\mathrm{R} \\
0.3 \\
0.3\end{array}$ & $\begin{array}{l}\mathrm{R} \\
\mathrm{R} \\
\mathrm{R} \\
\mathbf{1 2} \\
\mathrm{R} \\
\mathrm{R} \\
\mathrm{R} \\
\mathrm{R} \\
\mathrm{R} \\
\mathrm{R}\end{array}$ & $\begin{array}{c}\mathbf{R} \\
\mathbf{R} \\
\mathbf{R} \\
175 \\
125 \\
300 \\
\mathbf{R} \\
\mathbf{R} \\
\mathbf{R} \\
\mathbf{R}\end{array}$ & $\begin{array}{c}4.83 \\
42.0 \\
43.8 \\
7.11 \\
7.00 \\
4.10 \\
4.51 \\
3.10 \\
5.03 \\
4.10\end{array}$ & $\begin{array}{l}3.7 \\
3.0 \\
3.7 \\
3.8 \\
3.8 \\
2.1 \\
3.8 \\
3.7 \\
3.0 \\
2.3\end{array}$ & $\begin{array}{l}0.40 \\
4.75 \\
3.73 \\
0.58 \\
0.58 \\
0.19 \\
0.37 \\
0.26 \\
0.32 \\
0.31\end{array}$ & $\begin{array}{l}3.2 \\
2.2 \\
3.2 \\
3.5 \\
3.4 \\
3.4 \\
3.3 \\
3.4 \\
3.5 \\
2.1\end{array}$ \\
\hline
\end{tabular}

in which $f_{c}^{\prime}=$ uniaxial compressive strength; $f_{t}^{\prime}=$ uniaxial tensile strength; $f_{b c}^{\prime}=$ biaxial compressive strength; $f_{c}^{s}=$ pure shear strength; $\sigma_{r}=$ residual stress for very large uniaxial compressive strain; and $\tau_{r}=$ residual stress for very large shear strain at $\sigma_{V}=0$. The transformations according to (24) do not change these ratios. These ratios can be changed only by adjusting material parameters other than $E$ and $k_{1}$.

Parameters $k_{4}$ and $k_{5}$ can be determined exclusively from the data on hydrostatic compression tests. Taking the logarithm of $(20 a)$, the equation can be reduced to a linear regression plot, and thus parameters $k_{4}$ and $k_{5}$ can be obtained by fitting the data on the hydrostatic compression test alone, separately from all the other parameters (because the value of $\varepsilon_{V}$ for hydrostatic compression is the same for all microplanes and $\varepsilon_{D}$ $=\varepsilon_{M}=\varepsilon_{L}=0$ for all microplanes). The softening tail in uniaxial compression can be lengthened by increasing $c_{2}$ while reducing $k_{1}$ a little, and for tension by reducing $k_{3}$ while reducing $k_{1}$ a little. The ratio of the tensile to compressive strength can be increased by reducing $c_{4}$ or $k_{3}$. The ratio of the strength in pure shear to the uniaxial compressive strength can be increased by increasing $k_{2}$ while reducing $c_{4}$ or $k_{3}$ a little.

Eq. (23) for shear represents a linear friction law with cohesion and friction angle [Fig. 3(e)], which is a physically reasonable and simple way to handle inelastic response in shear (the cohesion stress is given by $E k_{1} k_{2}$, which is the frictional stress at $\sigma_{N}=0$ ). The stress-strain boundaries [Fig. 3(d)] in the plane $\left(\varepsilon_{T}, \sigma_{T}\right)$ are horizontal, which means they degenerate into a yield condition, unlike $(20)-(22)$. The frictional concept is particularly appropriate for describing the shear resistance of concrete that has been reduced by large strains to loose gravel.

If the present microplane is applied to extremely high pressures, as for example in impact problems, it seems that the straight line in Fig. 3(e) should be extended to the left by the curved dashed line approaching a horizontal. This would bring about a drop of friction (and dilatancy), known to exist at very high pressures. A possible simple formula could be $\sigma_{T}=$ $\left\langle\left(E k_{1} k_{2}-k_{3} \sigma_{N}\right) /\left(1-\sigma_{N} / k_{6}\right)\right\rangle$ with additional parameter $k_{6}$. The value of $k_{6}$ can be chosen large enough so that this formula would be very close to the straight line for the tests used to calculate the present model.

Dilatancy due to frictional shear deformation need not be introduced into the microplane frictional shear law. The microplane model automatically exhibits shear dilatancy due to the normal strains on microplanes inclined to the direction of shear slip (Bažant and Gambarova 1984).

The dependence of $c$ on $\sigma_{V}$ spoils the possibility of a completely explicit calculation of $\sigma_{i j}$ from given $\varepsilon_{i j}$. If this dependence were omitted, the correct ratio of biaxial to uniaxial compression strengths could not be obtained. The reason is that biaxial compression differs from uniaxial tension only by a hydrostatic stress state, and, to distinguish properly between these two stress states, $c$ cannot be independent of $\sigma_{V}$.

\section{GENERALIZATION TO MODERATELY LARGE FINITE STRAIN}

Generalization to finite strain is both a tensorial invariance problem and a constitutive problem. The generalization is accomplished by replacing the small strain tensor $\varepsilon_{y /}$ in the present model with the finite strain tensor $\varepsilon_{y}$. The transformation tensor $F_{y j}$ must be supplied as input to the constitutive subroutine.

However, there is an ambiguity: one has infinitely many possible finite strain expressions to choose from. The simplest choice is the Green-Lagrange strain tensor

$$
\varepsilon_{y}=\frac{1}{2}\left(F_{k d} F_{\forall}-\delta_{b j}\right)=\frac{1}{2}\left(u_{i, j}+u_{j, i}+u_{k, i} u_{k, j}\right)
$$

where $F_{u}=u_{i, j}+\delta_{i j} ; u_{i, j}=$ gradient of displacements $u_{i}$; and the subscripts preceded by a comma denote partial derivatives. These derivatives represent the derivatives with respect to the initial coordinates $X_{i}$ of material points (Lagrangian coordinates); i.e., $u_{i, j}=\partial u_{i} / \partial X_{j}$.

An important question arises for constitutive laws such as the present one, for which the strain tensor $\varepsilon_{i j}$ needs to be decomposed into volumetric and deviatoric finite strain tensors $\varepsilon_{V_{l j}}$ and $\varepsilon_{D_{l j}}$. At finite strain, $\varepsilon_{k t} / 3$ does not give the volume change exactly, and so generally $\varepsilon_{V_{i j}} \neq \varepsilon_{k k} / 3$ (this is so for any choice of finite strain tensor). But for applications involving high pressures, the volume change must be calculated exactly.

The importance of correct volumetric-deviatoric split may be illustrated by an example. Consider the case $F_{1,1}=0.50$, $F_{2,2}=1.25, F_{3,3}=1.55$, and $F_{2,1}=-0.55$, with all other $F_{i, j}=$ 0 . In this case, we obtain from (26): $\varepsilon_{11}=-0.2238, \varepsilon_{22}=$ $0.2813, \varepsilon_{33}=0.7013$, and $\varepsilon_{12}=\varepsilon_{21}=-0.3438$, with all other $\varepsilon_{i j}=0$. The trace is $\varepsilon_{k k}=0.7588$. The relative volume change $\left(V_{0}+\Delta V\right) / V_{0}=\operatorname{det} \mathbf{F}-1=-0.0313$, which is very different from $\varepsilon_{k k}$.

The volumetric-deviatoric split for Green-Lagrange finite strain $\varepsilon_{d}$ has recently been analyzed by Bažant (1994b). Let us now briefly review this analysis. It is helpful to begin by recalling the derivation of $\varepsilon_{i j}$. Let $X_{i}$ and $x_{i}=$ initial and final coordinates of material points, and consider a line segment $d X_{i}$ transforming to $d x_{i}$. The strain tensor $\varepsilon_{j}$ is defined by setting $d x_{k} d x_{k}-d X_{k} d X_{k}=2 \varepsilon_{i j} d X_{i} d X_{j}$. Substituting $x_{i}=X_{i}+u_{i}, d x_{k}=$ $x_{k, i} d X_{i}$ (where $x_{k, l}=\partial x_{k} / \partial X_{i}$ ), one gets

$$
\begin{aligned}
& 2 \varepsilon_{j} d X_{i} d X_{j}=x_{k, i} d X_{i} x_{k, j} d X_{j}-d X_{i} d X_{j} \\
& =\left[\left(X_{k}+u_{k}\right)_{i}\left(X_{k}+u_{k}\right)_{, j}-\delta_{i j}\right] d X_{l} d X_{J}
\end{aligned}
$$

Since this must hold for any $d X_{i}$, and $X_{k, i}=\partial X_{k} / \partial X_{i}=\delta_{k i}$, one has $2 \varepsilon_{j}\left(\delta_{k i}+u_{k, i}\right)\left(\delta_{k j}+u_{k, j}\right)-\delta_{i j}=u_{k, i}+u_{k, j}+u_{k, i} u_{k, j}$, which yields (26).

Proceeding similarly, we now imagine that a small material element is first subjected to a strictly volumetric (isotropic) 
expansion (i.e., same expansion in all directions), in which point $X_{i}$ moves to $\xi_{i}=X_{i}+u_{i}^{\prime}$ and line segment $d X_{i}$ transforms to line segment $d \xi_{i}$. Second, the element is transformed by deformation at no change of volume, followed by rigid body rotation (in which the volume change is also zero). In the second transformation, the point at coordinates $\xi_{i}$ moves to $x_{i}$ $=X_{i}+u_{i}$, and segment $d \xi_{i}$ transforms to $d x_{i}$. Let the volume change be characterized by engineering (or Biot) strain $\varepsilon_{0}$ defined so that $d \xi_{i}=\left(1+\varepsilon_{0}\right) d X_{i}$. The relative volume change is $\left(V_{0}+\Delta V\right) / V_{0}=J=\operatorname{det} F_{u}=\left(1+\varepsilon_{0}\right)^{3}$, where $J=$ Jacobian of transformation from $X_{i}$ to $x_{i} ; V_{0}=$ initial volume of small material element; and $\Delta V=$ its volume increase. Therefore

$$
\varepsilon_{0}=\left(\operatorname{det} F_{0}\right)^{1 / 3}-1
$$

Let us now denote $u_{i}^{\prime \prime}=x_{i}-\xi_{i}=$ displacements during the second transformation with zero volume change. Noting that $\partial \xi_{k} / \partial \xi_{k}=\delta_{k t}=$ Kronecker delta, we may write $d x_{k}=d\left(\xi_{k}+\right.$ $\left.u_{k}^{\prime \prime}\right)=\left[\partial\left(\xi_{k}+u_{k}^{\prime \prime}\right) / \partial \xi_{i}\right] d \xi_{i}=\left(\delta_{k i}+\partial u_{k}^{\prime \prime} / \partial \xi_{i}\right) d \xi_{i}$. Now, upon substituting $d \xi_{i}=\left(1+\varepsilon_{0}\right) d X_{i}$, we get $d x_{k} d x_{k}=\left(\delta_{k j}+\partial u_{k}^{\prime \prime}\right)$ $\left.\partial \xi_{i}\right) d \xi_{i}\left(\delta_{k j}+\partial u_{k}^{\prime \prime} / \partial \xi_{j}\right) d \xi_{j}$, or

in which

$$
d x_{k} d x_{k}=\left(\delta_{i j}+2 d_{i j}\right)\left(1+\varepsilon_{0}\right)^{2} d X_{i} d X_{j}
$$

$$
d_{i j}=\frac{1}{2}\left(\frac{\partial u_{j}^{\prime \prime}}{\partial \xi_{i}}+\frac{\partial u_{i}^{\prime \prime}}{\partial \xi_{j}}+\frac{\partial u_{k}^{\prime \prime}}{\partial \xi_{i}} \frac{\partial u_{k}^{\prime \prime}}{\partial \xi_{j}}\right)
$$

represents the Green-Lagrange finite strain tensor for the deviatoric transformation taken alone. The total change of length of the line segment may now be expressed as follows (Bažant 1994b):

$$
\begin{aligned}
2 \varepsilon_{i j} d X_{i} d X_{j} & =d x_{k} d x_{k}-d X_{k} d X_{k} \\
& =\left[\left(1+\varepsilon_{0}\right)^{2}\left(\delta_{j}+2 d_{j}\right)-\delta_{j}\right] d X_{i} d X_{j} \\
& =\left\{\left[\left(1+\varepsilon_{0}\right)^{2}-1\right] \delta_{i j}+2\left(1+\varepsilon_{0}\right)^{2} d_{i j}\right] d X_{i} d X_{J} \\
& =2\left(\varepsilon_{v_{j}}+\varepsilon_{D_{i j}}\right) d X_{i} d X_{J}
\end{aligned}
$$

in which we denoted

$$
\varepsilon_{D_{V}}=\left(1+\varepsilon_{0}\right)^{2} d_{i j} ; \quad \varepsilon_{V_{i j}}=\varepsilon_{V} \delta_{v} ; \quad \varepsilon_{V}=\varepsilon_{0}+\frac{1}{2} \varepsilon_{0}^{2}
$$

Comparing the first and last expressions in (31-34), we find

$$
\varepsilon_{D_{y}}=\varepsilon_{y}-\varepsilon_{v_{y}}
$$

Here $\varepsilon_{D_{j}}=$ additive deviatoric finite strain tensor; and $\varepsilon_{V_{j}}=$ Green-Lagrange volumetric finite strain tensor, which is the same as the Green-Lagrange finite strain tensor for the initial volumetric transformation taken alone.

The basic requirements for $\varepsilon_{V_{j}}$ and $\varepsilon_{D_{j}}$ to be volumetric and deviatoric tensors is that $\varepsilon_{v_{j}}$ must vanish for purely deviatoric deformation $\left(\varepsilon_{0}=0\right)$ and $\varepsilon_{D_{i j}}$ must vanish for purely volumetric deformation $\left(u_{i}^{\prime \prime}=0\right)$. These requirements are obviously satisfied. However, $\varepsilon_{D_{i j}}$ is not a purely volumetric strain measure because it depends on $\varepsilon_{0}$. This dependence is nevertheless practically unimportant because concrete cannot undergo volumetric strains more than about 3\% [which is the normal strain value at extreme hydrostatic pressure equal to $-300,000$ psi; see Bayant et al. (1986)]. Consequently, $\varepsilon_{0}$ cannot change the value of $\varepsilon_{D_{V}}$ by more than about $6 \%$, and for typical pressures not more than about $0.5 \%$.

An interesting point to note is that $\operatorname{det} A_{j,}$ in which $\left[A_{j}\right]^{2}=$ $\left[\delta_{j}+2 \varepsilon_{D_{y}}\right]$, is generally not 1 . The reason is that tensor $A_{i j}$ looks as the transformation tensor corresponding to $\varepsilon_{D_{i j}}$ only formally. To check the volume change corresponding to $\varepsilon_{D_{i j}}$, one needs to calculate it by the multiplicative decomposition.

The new result in (35)-(37) (Bažant 1994b) shows that, for the Green-Lagrange strain tensor, an additive decomposition into volumetric and deviatoric finite strains is possible. This additive property is advantageous for constitutive laws with the volumetric-deviatoric split and for our microplane model in particular. The additive split can also be achieved for other finite strain tensors [for example, Biot strain tensor or the logarithmic (Hencky) strain tensor; e.g., Bazant and Cedolin (1991), section 11.1), but is more complicated. The logarithmic strain tensor is the only case for which the additive volumetric-deviatoric split is pure (Bažant 1994b, 1995), i.e., $\varepsilon_{D_{y}}$ is independent of $\varepsilon_{0}$, but as has already been explained, this is unimportant for concrete.

The logarithmic strain tensor, however, has the advantage that it is the only strain measure for which the difference $\varepsilon_{i C}^{A}-\varepsilon_{v_{B}}^{A}$ of the finite strains at states $B$ and $C$ referred to natural state $A$ is the finite strain $\varepsilon_{i C}^{\mathrm{B}}$ at state $C$ referred to state $B$. This fact greatly simplifies the generalization of small-strain constitutive relation to very large strains. For the Green-Lagrange finite strain tensor, the proper generalization of a smallstrain constitutive relation to very large finite strains, of the order of $100 \%$, has been found next to impossible to figure out [because of the quadratic terms in (26)]. For this reason, the present generalization to finite strain must be restricted to moderately large finite strains of the order of $10 \%$. It is planned to extend the formulation to very large strains of concrete, of the order of $100 \%$, by using the logarithmic strain tensor (Bažant 1994b) (deviatoric strains of such magnitude can occur under extremely large hydrostatic pressures).

Because of the additivity of volumetric and deviatoric strain tensors in (36), the microplane normal strain $\varepsilon_{N}$ can be split as follows:

$$
\varepsilon_{D}=\varepsilon_{N}-\varepsilon_{v}=N_{i j} \varepsilon_{i j}-\varepsilon_{0}-(1 / 2) \varepsilon_{0}^{2}
$$

(although $\varepsilon_{V} \neq \varepsilon_{k k} / 3$ ). The calculation of stress tensor $\sigma_{i j}$ from the microplane stresses $s_{i j}$ according to (9) remains valid.

If the finite strain tensor used as the input of the constitutive model is the Green-Lagrange strain, the output is the so-called second Piola-Kirchhoff (2PK) stress tensor. But often the stress tensor used in the equations of motion or equilibrium of the structure is the Cauchy stress tensor $S_{v}$ (also called the true stress, representing the actual forces acting on a small unit cube cut out from the deformed material)

$$
S_{i j}=\left(\delta_{i k}+u_{i, k}\right)\left(\delta_{j m}+u_{j, m}\right) \sigma_{k m} / J
$$

See, for example, Eqs. 11.2.9 and 11.2.13 in Bazant and Cedolin (1991).

A change in the choice of finite strain tensor is manifested by a change of the associated objective stress rate (Bažant and Cedolin 1991, Eq. 11.3.19). But this, in turn, has been shown equivalent to a change in the tangential stiffness moduli of the material (Bažant 1971; Bažant and Cedolin 1991, Eq. 11.4.4). Therefore, a change in the choice of finite strain tensor must be equivalent to a certain change in the functions describing the constitutive properties (Bažant 1994b). Any finite strain tensor $\tilde{\mathbf{\varepsilon}}$ may be expressed as $\tilde{\boldsymbol{\varepsilon}}=\mathbf{G}(\mathbf{\varepsilon})$, where $\mathbf{G}=$ a tensorvalued function of Green-Lagrange strain tensor $\varepsilon$ of components $\varepsilon_{y}$. Most generally, function $\mathbf{G}$ may be defined by the polar decomposition of tensor

$$
\widetilde{\varepsilon}_{j k}=G_{j}(\varepsilon)=\sum_{i=1}^{3} g\left[\varepsilon_{(n)}\right] n_{j}^{(0)} n_{k}^{(0)}
$$

where $n_{j}^{(n)}=$ direction cosines of the principal axes of tensor $\varepsilon$ (and also of $\tilde{\boldsymbol{\varepsilon}}$ ); $\varepsilon_{(i)}=$ principal Green-Lagrange strains; and $g(\varepsilon)=$ any continuous monotonically increasing function such that $g(0)=0$ and $g^{\prime}(0)=1$. Eq. (39) is analogous to the ex- 
pression given by Ogden (1984) and Rice (1993) in terms of the principal stretches $\lambda_{(i)}=\sqrt{1+2 \varepsilon_{(i)}}$. As recognized by Hill (1968), the stress tensor $\tilde{\boldsymbol{\sigma}}$ that is conjugate to $\tilde{\boldsymbol{\varepsilon}}$ must be defined by $\tilde{\boldsymbol{\sigma}}: \delta \tilde{\boldsymbol{\varepsilon}}=\sigma: \delta \varepsilon$. This variational equation, which requires that the work of the conjugate stresses $\tilde{\boldsymbol{\sigma}}$ on any strain variation must be the same for any choice of finite strain tensor, may be rearranged as $[\tilde{\boldsymbol{\sigma}}:(\partial \tilde{\boldsymbol{\varepsilon}} / \partial \varepsilon)-\sigma]: \delta \varepsilon=0$. This implies that $[\cdots]=0$, or

$$
\boldsymbol{\sigma}=\tilde{\boldsymbol{\sigma}}: \frac{\partial \mathbf{G}(\boldsymbol{\varepsilon})}{\partial \boldsymbol{\varepsilon}} \text { or } \sigma_{i j}=\tilde{\boldsymbol{\sigma}}_{k l} \frac{\partial G_{k l}(\varepsilon)}{\partial \varepsilon_{i j}}
$$

Here, $\tilde{\sigma}_{k t}$ and $G_{k l}=$ components of tensor $\tilde{\boldsymbol{\sigma}}$ and $\mathbf{G}$; and the colon refers to a doubly contracted tensorial product. Now, the small-strain constitutive law, which we write as $\mathbf{G}=\varphi(\varepsilon)$, defined by tensor-valued function $\tilde{\varphi}$, can be generalized by (1) reinterpreting the small (or linearized) strain as finite strain $\tilde{\boldsymbol{\varepsilon}}$, representing anyone of the possible finite strain tensors; and (2) at the same time reinterpreting stress $\sigma$ as the stress $\tilde{\sigma}$ conjugate to $\tilde{\boldsymbol{\varepsilon}}$. Thus the constitutive law generalized to finite strain takes in general the form $\tilde{\sigma}=\varphi(\bar{\varepsilon})$. So, according to (40)

$$
\boldsymbol{\sigma}=\varphi[\mathbf{G}(\varepsilon)]: \frac{\partial \mathbf{G}(\varepsilon)}{\partial \varepsilon}=\mathbf{f}(\varepsilon)
$$

Function $f$ must asymptotically coincide with $\varphi$ for small strains (i.e., up to linear terms in $\varepsilon_{i j}$ ).

So we see that the constitutive law given by tensor-valued function $\varphi$ that relates some strain tensor $\tilde{\varepsilon}_{i j}$ to its conjugate stress tensor $\tilde{\sigma}_{i j}$ must be equivalent to the constitutive law given by a certain other tensor-valued function $f$ relating the 2PK stress tensor $\sigma_{i j}$ to the Green-Lagrange strain tensor $\varepsilon_{i j}$. Therefore, it is necessary to consider transformations of the constitutive law that are approximately equivalent to the transformation from $\varphi$ to $f$ due to other possible choices of the finite strain tensor.

The $6 \times 6$ matrix of the components of fourth-rank tensor $\partial \mathbf{G}(\boldsymbol{\varepsilon}) / \partial \boldsymbol{\varepsilon}$ can be calculated for any choice of finite strain (Bažant 1994b), but it is not easy and we better avoid it. It is better to define function $f(\varepsilon)$ directly. It is convenient to do so as an implicit function of Green-Lagrange strain $\varepsilon$, in the form $f(\varepsilon)=\varphi[\psi(\varepsilon)]$ where $\varphi$ defines, as before, the small strain constitutive law, and $\psi(\varepsilon)$ is an arbitrary tensor-valued function of $\varepsilon$ such that the linear term of its Taylor series expansion coincides with $\varepsilon$, i.e., $\psi(\varepsilon) \approx \varepsilon$ up to linear terms. Thus function $\psi(\varepsilon)$ represents the combined effect of replacing $\varepsilon$ by $\mathbf{G}(\boldsymbol{\varepsilon})$ and applying the transformation $\partial \mathbf{G} / \partial \boldsymbol{\varepsilon}$ in (41).

In the context of the microplane model, the transformation according to function $\psi$ may be more conveniently introduced on the microplane level, i.e., as transformations $\psi_{N}\left(\varepsilon_{N}\right)$ and $\psi_{T}\left(\varepsilon_{T}\right)$ for the microplane normal strain $\varepsilon_{N}$ and the microplane normal strains $\varepsilon_{T}=\varepsilon_{M}$ and $\varepsilon_{T}=\varepsilon_{L}$. Limiting this transformation to terms up the third order in Taylor series expansion, we may carry out the replacements

$$
\begin{gathered}
\varepsilon_{N} \leftarrow \psi_{N}\left(\varepsilon_{N}\right)=\varepsilon_{N}\left[1+\frac{p+(2 p+q) \varepsilon_{N}}{1+2 \varepsilon_{N}} \varepsilon_{N}\right] \\
\varepsilon_{T} \leftarrow \psi_{T}\left(\varepsilon_{T}\right)=\varepsilon_{T} \frac{1+\langle r\rangle \varepsilon_{T}^{2}}{1+\langle-r\rangle \varepsilon_{T}^{2}}
\end{gathered}
$$

in which $\varepsilon_{N} \in(-0.5, \infty) ; \varepsilon_{T} \in(-\infty, \infty)$; and $p, q$, and $r=$ additional empirical constitutive parameters for the generalization to large strains, which can be determined only by fitting large-strain data. They should not be so large as to significantly alter the response within the range of small strain data. Because, for the case of volumetric strain tensor $\varepsilon_{i j}=\delta_{j j} \varepsilon_{V}$ (or $\varepsilon_{D}=0$ ), the transformations of $\varepsilon_{N}$ and $\varepsilon_{V}$ must coincide, it is also necessary to make the replacement $\varepsilon_{V} \leftarrow \psi_{N}\left(\varepsilon_{V}\right)$, where $\psi_{N}=$ same function as in (42a). After that, one may calculate $\varepsilon_{D}=\varepsilon_{N}-\varepsilon_{V}$.

For small $\varepsilon_{N}$ and $\varepsilon_{T},(42 a)$ and (42b) are equivalent, up to the third-degree terms in $\varepsilon_{N}$ and $\varepsilon_{T}$, to

$$
\psi_{N}\left(\varepsilon_{N}\right) \approx \varepsilon_{N}+p \varepsilon_{N}^{2}+q \varepsilon_{N}^{3} \text { and } \psi_{T}\left(\varepsilon_{T}\right) \approx \varepsilon_{T}+r \varepsilon_{T}^{3}
$$

The forms in (42a) and (42b), however, are preferable. They have been set up so as to satisfy the following conditions for arbitrarily large strains: (1) function $\psi_{T}\left(\varepsilon_{T}\right)$ must never be negative, and indeed, by virtue of using $\langle\cdots\rangle,(42 b)$ is nonnegative, while $(43 b)$ for $q<0$ becomes negative for sufficiently large $\varepsilon_{T} ;(2)$ function $\psi_{N}\left(\varepsilon_{N}\right)$ must approach $-\infty$ for $\varepsilon_{N} \rightarrow$ $-1 / 2$ because in that case a finite line segment in the direction normal to the microplane gets compressed into a point. Note also that the quadratic term must be absent from function $\psi_{T}$ because this function must be antisymmetric (otherwise shearing in one direction would give a different response than shearing in the opposite direction).

Functions $F_{V}, F_{D}, F_{N}$, and $F_{T}$ in (20)-(23) have been selected in a form that is not unreasonable for large strains. Although $F_{N}$ vanishes for $\varepsilon_{N} \rightarrow \infty$ and $F_{D}$ for $\varepsilon_{D} \rightarrow \infty$, function $F_{V}$ provides increasing resistance to large volume decrease and function $F_{T}$ provides frictional shear resistance of the material depending on the magnitude of compressive $\sigma_{N}$. This approximately describes the behavior of gravel-like rubble, to which concrete may be reduced at very large shear strain.

Even when concrete is intersected by a continuous crack, the microplane model provides resistance to shear along the plane of the crack, depending on strain and stress normal to the crack. This is due to the fact that a shear along such a crack is accompanied by compressive strain in $45^{\circ}$ inclined direction, and those strains are resisted by the microplane model. This resistance further causes the microplane model to exhibit dilatancy due to shear of cracks (Bazant and Gambarova 1984).

\section{CALCULATION OF FINITE STRAINS FROM VELOCITY GRADIENTS AND SPINS}

In large explicit finite-element programs for finite strain, typically the variables available at the beginning of each time step are the deformation rate tensor $D_{i}$ and the spin tensor $W_{i j}$. From this, the velocity gradient tensor $L_{j}=D_{i j}+W_{i j}$. To calculate $\varepsilon_{i j}$, one needs the transformation $F_{i j}=\delta_{i j}+u_{i j}=$ $\partial x_{i} / \partial X_{i}$, where $X_{i}$ and $x_{i}=$ initial and current coordinates of material point $\left(X_{i}=x_{i}+u_{i}\right)$; and $L_{i j}=\partial v_{i} / \partial x_{j}=\partial \dot{x}_{i} / \partial x_{j}=\partial \dot{u}_{i}$ $/ \partial x_{i}$, where $v_{i}=\dot{x}_{i}=\dot{u}_{i}=$ material point velocity. Now, $\dot{F}_{i j}=$ $\partial \dot{x}_{i} / \partial X_{j}=\partial v_{i} / \partial X_{j}=\left(\partial v_{i} / \partial x_{k}\right)\left(\partial x_{k} / \partial X_{j}\right)=L_{i k} F_{k j}$. Therefore, the increment of $F_{i}$ during the time step $\Delta t$ is

$$
\Delta F_{i j}=L_{i k} F_{k j} \Delta t
$$

with first-order accuracy in $\Delta t$ if $L_{i k}$ and $F_{k}$ refer to the beginning of time step. After updating $F_{i j} \leftarrow F_{i j}+\Delta F_{i j}$, one can calculate the new total Green-Lagrange finite strain as $\varepsilon_{i j}=$ $\left(F_{k i} F_{k j}-\delta_{i j}\right) / 2$.

If the step is iterated and $L_{i k} F_{k j}$ are reevaluated on the basis of the first iteration for the middle of the time step, then the accuracy in $\Delta t$ is of the second order. Second-order accuracy in $\Delta t$ can also be achieved during first iteration if the acceleration gradient $\dot{L}_{i j}=\partial \dot{v}_{i} / \partial X_{j}$ (or $D$ and $W$ ) at the beginning of the time step is calculated by the finite-element program. In that case one can use, with second-order accuracy in $\Delta t$, $\Delta F_{i j}=L_{i k} F_{k} \Delta t+F_{j}\left(\Delta t^{2} / 2\right)$ where $F_{j}=\left[\left(\partial v_{i} / \partial x_{k}\right)\left(\partial x_{k} / \partial X_{j}\right]=\right.$ $\left(\partial \dot{v}_{i} / \partial x_{k}\right)\left(\partial x_{k} / \partial X_{f}\right)+\left(\partial \dot{x}_{k} / \partial X_{j}\right)$ with $\partial \dot{x}_{k} / \partial X_{j}=\left(\partial v_{k} / \partial x_{m}\right)\left(\partial x_{m} /\right.$ $\left.\partial X_{j}\right)$, and so

$$
\Delta F_{j}=L_{i k} F_{k j} \Delta t+\left(L_{i k} L_{k m} F_{m j}+L_{i k} F_{k j}\right)\left(\Delta t^{2} / 2\right)
$$


Note that, at constant velocity, $L_{i k}$ vanishes but the term $L_{i k}$ $L_{k m} F_{m j}$ remains. Thus it might be useful to keep this term even if the value of $L_{i k}$ at the beginning of the time step is unavailable.

\section{SUMMARY}

The microplane model simplifies constitutive modeling because the stress-strain relation on the microplane level involves only a few stress and strain components, which have a clear physical meaning. The passage from elastic response to softening damage defined in terms of different variables is simplified by the concept of boundaries in the stress-strain space. The development of the general theoretical concept in this paper will be followed in the subsequent companion paper (Bažant et al. 1996) by comparisons with test data and a procedure to filter out unwanted localization from test data.

\section{APPENDIX I. SMOOTH TRANSITION TO BOUNDARY CURVES}

If we simply assume the response anywhere within the boundaries to be elastic, as given in the rate form by (10), the stress-strain path for the microplane will exhibit a sudden change of slope because the response cannot exceed the boundary curve. This kind of microplane behavior will cause rather abrupt changes of slope of the stress-strain relation on the macrolevel, although not as abrupt as on the microplane because different microplanes reach the boundary at different moments of loading. Experiments do not show such abrupt slope changes. Therefore, a remedy is desirable.

The sudden transition from elastic response to the response along the stress-strain boundary causes the peaks of the stressstrain curve to be rather sharp (as seen in Fig. 3 of Bažant et al.). The peaks can be smoothed out by introducing a transition curve between the elastic straight line and the boundary curve. The transition curve, however, cannot be defined as a simple function of strains because the elastic lines and boundary curves are functions of different components. A helpful idea is to define the transition implicitly, in terms of (1) the elastic stress value $\sigma^{e}$ for a given strain $\varepsilon$; and (2) the boundary curve value $\sigma^{b}$, both corresponding to the same strain $\varepsilon$. When $\sigma^{b} \gg \sigma^{c}>0$, the transition curve must nearly coincide with $\sigma^{e}$; when $\sigma^{b} \ll \sigma^{o}$, it must nearly coincide with $\sigma^{b}$; and when $\sigma^{e}=\sigma^{b}$, it must lie the farthest below both curves. These required properties can be achieved by the following formula for the transition curve:

$T\left(\sigma^{e}, \sigma^{b}\right)=\frac{\sigma^{b}+\sigma^{e}+\delta_{1}}{2}-\delta_{0} \ln \left(2 \cosh \frac{\sigma^{b}-\sigma^{e}-\delta_{1}}{2 \delta_{0}}\right)$

where $\sigma^{e}=\sigma_{D}^{e}, \sigma_{N}^{e}$, or $\sigma_{T}^{e} ; \sigma^{b}=\sigma_{D}^{b}, \sigma_{N}^{b}$, or $\sigma_{T}^{b}$; and $\delta_{1}$ and $\delta_{0}$ $=$ constants, which could be chosen as $\delta_{1}=0.10 f_{0}$ sign $\left(\sigma^{b}\right)$ and $\delta_{0}=0.24 f_{0}$ sign $\left(\sigma^{b}\right)$ with $f_{0}=f_{D}^{0}, f_{N}^{0}$, or $f_{T}^{0}$. For the volumetric boundary, no transition curve needs to be introduced because the slope change is mild.

For $\delta_{1}=0$, the transition curve would approach the elastic curve and the boundary curve asymptotically at $\pm \infty$ (this may be easily checked by noting that, for large $|x|, 2 \cosh x=$ $\exp |x|)$. But the response near the origin of stress-strain space must be exactly elastic. Therefore, the left-side asymptote of the transition curve needs to be shifted up by distance $\delta_{1}$. This causes the transition to intersect the elastic curve. By choosing a small enough $\delta_{1}$, the slope change at the intersection can be made small and acceptable.

Transition curve (46), however, complicates the computational algorithm in finite-element applications and doubles the number of stress variables that need to be stored. At the same time, the sharpness of the peaks of stress-strain relations is not important for most applications, compared to the magnitude of the peak and the area under the postpeak stress-strain curve. For this reason, (46) has not been included in the finite-element program.

Instead of (46), another formula of similar properties could also be introduced: $T\left(\sigma^{e}, \sigma^{b}\right)=\left\{\sigma^{b}+\sigma^{e}+\delta_{1}-\left[\left(\sigma^{b}-\right.\right.\right.$ $\left.\left.\left.\sigma^{2}-\delta_{1}\right)^{2}+\delta_{0}^{2}\right]^{1 / 2}\right\} / 2$. This formula would be faster to execute computationally. However, it approaches the elastic and boundary curves too slowly, much slower than (46).

\section{APPENDIX. REFERENCES}

Batdorf, S. B., and Budianski, B. (1949). "A mathematical theory of plasticity based on the concept of slip." Tech. Note No. 1871, Nat. Advisory Committee for Aeronautics, Washington, D.C.

Bažant, Z. P. (1971). "A correlation study of incremental deformations and stability of continuous bodies." J. Appl. Mech. Trans. ASME, 38, 919-928.

Bažant, Z. P. (1984). "Size effect in blunt fracture: Concrete, rock, metal." J. Engrg. Mech., ASCE, 110(4), 518-535.

Bažant, Z. P. (1993). "Concept of stress-strain boundaries for microplane model." Internal Res. Note, Dept. of Civ. Engrg., Northwestern Univ., Evanston, Ill.

Bažant, Z. P. (1994a). "Nonlocal damage theory based on micromechanics of crack interactions." J. Engrg. Mech., ASCE, 120(3), 593-617.

Bažant, Z. P. (1994b). "Finite-strain generalization of small-strain constitutive relations and additive volumetric-deviatoric split." Rep. 9412/403f, Dept. of Civ. Engrg., Northwestern University; Evanston, Ill., Int. J. Solids and Struct., 32(1996).

Bažant, Z. P. (1995). "Additive volumetric-deviatoric split of finite strain tensor and its implication for cracking models." Fracture mechanics of concrete structures: Proc., 2nd Int. Conf. on Fracture Mech. of Concrete and Concrete Struct. (FraMCoS-2), F. H. Whittmann, ed., Aedificatio Publishers, Freiburg, Germany, 515-534.

Bazant, Z. P., Bishop, F. C., and Chang, T.-P. (1986). "Confined compression tests of cement paste and concrete up to $300 \mathrm{ksi}$." J. Am. Concrete Inst., 33(4), 553-560.

Bažant, Z. P., and Cedolin, L. (1991). Stability of structures: Elastic, inelastic, fracture and damage theories (textbook and reference volume), Oxford University Press, New York, N.Y.

Bažant, Z. P., and Gambarova, P. (1984). "Crack shear in concrete: Crack band microplane model." J. Struct. Engrg., ASCE, 110(9), 20152035.

Bažant, Z. P., Jirásek, M., Xiang, Y., and Prat, P. C. (1994). “Microplane model with stress-strain boundaries and its identification from tests with localized damage." Proc., Computational Modeling of Concrete Struct. (EURO-C), H. Mang, et al., eds., Pineridge Press, Swansea, Wales, 255-261.

Bazant, Z. P., and Oh, B.-H. (1983). "Microplane model for fracture analysis of concrete structures." Proc., Symp. on Interaction of NonNuclear Munitions with Struc., held at U.S. Air Force Academy, Colorado Springs, Colo., 49-53.

Bažant, Z. P., and Oh, B.-H. (1985). "Microplane model for progressive fracture of concrete and rock." J. Engrg. Mech., ASCE, 111(4), 559582.

Bažant, Z. P., and Oh, B.-H. (1986). "Efficient numerical integration on the surface of a sphere." Zeitschrift fur angewandte Mathematik und Mechanik (ZAMM), Berlin, Germany, 66(1), 37-49.

Bažant, Z. P., and Ožbolt, J. (1990). "Nonlocal microplane model for fracture, damage, and size effect in structures." J. Engrg. Mech., ASCE, 116(11), 2484-2504.

Bažant, Z. P., and Prat, P. C. (1988a). "Microplane model for brittle plastic material. I: Theory." J. Engrg. Mech., ASCE, 114(10), 16721688.

Bažant, Z. P., and Prat, P. C. (1988b). "Microplane model for brittle plastic material: II. Verification." J. Engrg. Mech., ASCE, 114(10), $1689-1702$.

Bažant, Z. P., Xiang, Y., Adley, M. D., Prat, P. C., and Akers, S. A. (1996). "Microplane model for concrete. II: Data delocalization and verification." J. Engrg. Mech., ASCE, 122(3), 255-262.

Budianski, B., and Wu, T. T. (1962). "Theoretical prediction of plastic strains of polycrystals.", Proc., 4th U.S. Nat. Congr. of Appl. Mech., ASME, New York, N.Y., 1175-1185.

Carol, I., Bažant, Z. P., and Prat, P. C. (1991). "Geometric damage tensor based on microplane model." J. Engrg. Mech., 117(10), 2429-2448.

Carol, I., Prat, P. C., and Bažant, Z. P. (1992). "New explicit microplane model for concrete: Theoretical aspects and numerical implementation." Int. J. Solids and Struct., 29(9), 1173-1191.

Cofer, W. F. (1992). "Implementation of the nonlocal microplane con- 
crete model within an explicit dynamic finite element program." Appl. Mech. Rev., 45(3), part 2, S132-S139.

Cofer, W. F., and Kohut, S. W. (1994). "A general nonlocal microplane concrete material model for dynamic finite element analysis." Comp. and Struct., 53(1), 189-199.

Hasegawa, T., and Bažant (1993). "Nonlocal microplane concrete model with rate effect and load cycles. I. General formulation." J. Mat. in Civ. Engrg., ASCE, 5(3), 372-410.

Hill, R. (1965). "Continuum micromechanics of elastoplastic polycrystals." J. Mech. and Phys. in Solids, 13, 89-101.

Hill, R. (1966). "Generalized constitutive relations for incremental deformations of metal crystals by multi-slip." J. Mech. and Phys. of Solids, 14, 95-102.

Hill, R. (1968). "On constitutive inequalities for simple materials." $J$. Mech. and Phys. of Solids, 16, 229-242, 315-322.

Jirásek, M. (1993). "Modeling of fracture and damage in quasi-brittle materials," PhD dissertation, Northwestern Univ., Evanston, Ill.

Kröner, E. (1961). "Zur Plastischen Verformung des Vielkristalls." Acta Metullurgica, 9(Feb.), 155-161.

Lin, T. H., and Ito, M. (1965). "Theoretical plastic distortion of a polycrystalline aggregate under combined and reversed stresses." J. Mech. and Phys. of Solids, 13, 103-115.

Ogden, R. W. (1984). Non-linear elastic deformations. Ellis Horwood, Ltd., Chichester, U. K. and John Wiley \& Sons, Chichester, U.K.

Lin, T. H., and Ito, M. (1966). "Theoretical plastic stress-strain relationship of a polycrystal." Int. J. Engrg. Sci., 4, 543-561.

Ožbolt, J., and Bažant, Z. P. (1992). "Microplane model for cyclic triaxial behavior of concrete and rock." J. Engrg. Mech., ASCE, 118(7), $1365-1386$.

Pande, G. N., and Sharma, K. G. (1981). "Implementation of computer procedures and stress-strain laws in geotechnical engineering." Proc., Symp. on Implementation of Comp. Procedures and Stress-Strain Laws in Geotech. Engrg., C. S. Desai and S. K. Saxena, eds., Acorn Press, Durham, N.C., 575-590.

Pande, G. N., and Sharma, K. G. (1982). "Multi-laminate model of clays-A numerical evaluation of the influence of rotation of the principal stress axis." Rep., Dept. of Civ. Engrg., Univ. Coll. of Swansea, U.K.

Pande, G. N., and Xiong, W. (1982). "An improved multi-laminate model of jointed rock masses." Proc., Int. Symp. on Numerical Models in Geomech., R. Dungar, G. N. Pande, and G. A. Studder, eds., A. A. Balkema, Rotterdam, The Netherlands, 218-226.

Rice, J. R. (1970). "On the structure of stress-strain relations for timedependent plastic deformation of metals." J. Appl. Mech., 37(Sept.), $728-737$.

Rice, J. R. (1993). "Mechanics of solids." Encyclopedia Brittanica, 15th Ed., 23, 737-747, 773.

Stroud, A. H. (1971). Approximate calculation of multiple integrals. Prentice-Hall, Englewood Cliffs, N.J.

Taylor, G. I. (1938). "Plastic strain in metals." J. Inst. Metals, 62 , 307-324.

Zienkiewicz, O. C., and Pande, G. N. (1977). "Time-dependent multilaminate model of rocks - A numerical study of deformation and failure of rock masses." Int. J. Numerical and Analytical Methods in Geomech., 1, 219-247. 